\title{
DESIGN OF UNKNOWN INPUT FRACTIONAL-ORDER OBSERVERS FOR FRACTIONAL-ORDER SYSTEMS
}

\author{
Ibrahima N'DOYE*, Mohamed DAROUACH ${ }^{* *}$, Holger VOOS *, Michel ZASADZINSKI ** \\ ${ }^{*}$ Faculty of Science, Technology and Communication (FSTC) \\ University of Luxembourg, 6, rue Richard Coudenhove-Kalergi, L-1359 Luxembourg \\ e-mail: ibrahima.ndoye@uni.lu \\ ** Research Center for Automatic Control of Nancy (CRAN UMR, 7039, CNRS) \\ University of Lorraine, IUT de Longwy, 186 rue de Lorraine, 54400 Cosnes et Romain, France
}

\begin{abstract}
This paper considers a method of designing fractional-order observers for continuous-time linear fractional-order systems with unknown inputs. Conditions for the existence of these observers are given. Sufficient conditions for the asymptotical stability of fractional-order observer errors with the fractional order $\alpha$ satisfying $0<\alpha<2$ are derived in terms of linear matrix inequalities. Two numerical examples are given to demonstrate the applicability of the proposed approach, where the fractional order $\alpha$ belongs to $1 \leq \alpha<2$ and $0<\alpha \leq 1$, respectively. A stability analysis of the fractional-order error system is made and it is shown that the fractional-order observers are as stable as their integer order counterpart and guarantee better convergence of the estimation error.
\end{abstract}

Keywords: fractional calculus, fractional-order systems, fractional-order observers, existence condition, linear matrix inequality, unknown input, stability.

\section{Introduction}

State estimators or observers have been widely used in control and signal processing in the last few decades. They are of theoretical interest and have also led to many successful applications, e.g., in failure detection and fault diagnosis problems or in chaotic synchronization and secure communications (Darouach et al., 1994; Boutayeb et al., 2002).

The problem of functional observer design was related to constrained or unconstrained Sylvester equations (Tsui, 1985; Van Dooren, 1984). To solve this problem, many authors have generally proposed to transform the initial system to an equivalent one (by using some regular transformations) of reduced order and to design an observer for this system. Necessary and sufficient conditions for the existence of these observers for integer order linear systems were given by Darouach (2000), Watson and Grigoriadis (1998), as well as Trinh and Fernando (2012). The observers for systems with unknown inputs are of great interest in failure detection and the control of systems in the presence of disturbances (Darouach et al., 1994; Trinh and Fernando, 2012).

The use of non-integer orders in systems theory is not a new concept-many successful applications of differintegration have been proposed in the last two decades. The field of automatic control systems is also influenced by this new perspective of operators with a significant number of contributions, e.g., fractional-order variants of Proportional Integral Derivative (PID) controllers (Podlubny, 1999; Monje et al., 2010; Caponetto et al., 2010). Fractional-order systems have been studied by many authors in engineering sciences from an application point of view (see, e.g., the works of Podlubny (1999), Hilfer (2001), Kilbas et al. (2006) or Kaczorek (2011a) and the references therein). Many systems can be described with the help of fractional derivatives: electromagnetic systems (Heaviside, 1971; Engheta, 1996), dielectric polarization (Sun et al., 1984), a supercapacitor (Kaczorek, 2011b), viscoelastic systems (Bagley and Calico, 1991; Rossikhin and Shitikova, 1997), and chaotic synchronization or secure communications (Delshad et al., 2011).

The question of stability is crucial in control theory. In the field of fractional-order control systems, there are many challenging and unsolved problems related to stability theory such as robust stability, bounded 
input-bounded output stability, internal stability, etc. Some early results on the stability of fractional-order control systems were presented by Matignon (1996), Chen et al. (2006), Petráš et al. (2004) and Petrás̆ (2011).

One highly relevant but so far scarcely addressed problem is the design of observers for fractional-order systems. In the literature, there are only very limited reports on the estimation and compensation of disturbances (Chen et al., 2004). Recently, the observability and stability problems of linear fractional-order systems using a continuous frequency distribution have been presented by Sabatier et al. (2012) and Trigeassou et al. (2011), respectively. It is shown that the pseudo-state-space representation, usually encountered in the literature for fractional-order systems, can be used to design Luenberger-like observers that permit estimation of important variables in the system. Furthermore, the convergence of the observation was considered by Sabatier et al. (2012). For fractional-order nonlinear systems, a novel robust fractional-order sliding mode observer was presented by Dadras and Momeni (2011b), who also proposed a simple fractional-order observer design (Dadras and Momeni, 2011a). The problem of non-fragile observer design for a class of Lipschitz nonlinear factional-order systems using continuous frequency distribution was presented by Boroujeni and Momeni (2012), based on an indirect approach to Lyapunov stability to derive the stability conditions.

In this paper, a fractional-order observer design is presented to solve the problem of state reconstruction for fractional-order linear systems with unknown inputs. It is shown that the proposed observer guarantees the convergence of the state estimation errors. The fractional-order stability condition is exploited to analyze the stability of the estimation fractional-order error system. It ought to be mentioned that the proposed observer is as stable as its integer order counterpart, very simple and constructive for practical applications.

This paper is organized as follows. In Section 2 we provide some background on the fractional derivative, the stability and the detectability of fractional-order systems with the fractional order $0<\alpha<2$. In Section 3 , we formulate a condition for the existence and a functional observer design problem for linear fractional-order systems. Sufficient conditions for the asymptotical stability of observers with the fractional order $\alpha$ belonging to the ranges $0<\alpha \leq 1$ and $1 \leq$ $\alpha<2$ are presented in terms of linear matrix inequalities. Finally, two numerical examples are given to illustrate our proposed results.

Notation. $M^{T}$ is the transpose of $M, \operatorname{Sym}\{X\}$ is used to denote $X^{T}+X, \Sigma^{+}$is any generalized inverse of $\Sigma$ satisfying $\Sigma \Sigma^{+} \Sigma=\Sigma, \otimes$ stands for the Kronecker product and $D^{\alpha}$ represents the initialized $\alpha$-th order differintegration.

\section{Preliminary results}

In this section, we present some preliminary results on fractional derivative systems which will be used in the sequel of this paper.

Fractional-order differentiation is a generalization of integer-order one. Formulations of fractional-order derivatives fall into two main classes: the Riemann-Liouville derivative defined as (Podlubny, 1999)

$$
\begin{aligned}
& D^{\alpha} f(t)=\frac{1}{\Gamma(n-\alpha)} \frac{\mathrm{d}^{n}}{\mathrm{~d} t^{n}} \int_{0}^{t} \frac{f(\tau)}{(t-\tau)^{\alpha-n+1}} \mathrm{~d} \tau, \\
& n-1<\alpha<n \text {, }
\end{aligned}
$$

or the Caputo derivative defined as (Podlubny, 2002)

$$
\begin{aligned}
D^{\alpha} f(t)=\frac{1}{\Gamma(\alpha-n)} \int_{0}^{t} \frac{\frac{\mathrm{d}^{n} f(\tau)}{\mathrm{d} t^{n}}}{(t-\tau)^{\alpha-n+1}} \mathrm{~d} \tau, \\
n-1<\alpha<n,
\end{aligned}
$$

with $n \in \mathbb{N}$ and $\alpha \in \mathbb{R}^{+}$, where $\Gamma(\cdot)$ is the Gamma function defined by the integral

$$
\Gamma(z)=\int_{0}^{\infty} e^{-t} t^{z-1} \mathrm{~d} t .
$$

A physical interpretation of fractional derivatives and the solution of fractional differential equations are given by Podlubny (2002). Here and throughout the paper, only the Caputo definition is used since its Laplace transform allows the use of initial values of classical integer-order derivatives with clear physical interpretations. In the rest of this paper, $D^{\alpha}$ is used to denote the Caputo fractional derivative of order $\alpha$.

For numerical simulation of fractional-order systems, we can use the Grünwald-Letnikov method (Petráš, 2010; 2011) based on the Adams-Bashforth-Moulton type predictor-corrector scheme (Deng, 2007). The method is suitable for Caputo's derivative because it just requires the initial conditions and has a clear physical meaning for unknown functions. The relation for the explicit numerical approximation of the $\alpha$-th derivative at the points $k h$ (see also Dorckák, 1994; Podlubny, 1999; Petráš, 2010; 2011) is given by

$$
{ }_{\left(k-L_{m} / h\right)} D_{k h}^{\alpha} f(t) \approx h^{-\alpha} \sum_{j=0}^{k}(-1)^{j}\left(\begin{array}{c}
\alpha \\
j
\end{array}\right) f_{k-j},
$$

where $L_{m}$ is the memory length, $h$ is the time step of the calculation and $(-1)^{j}\left(\begin{array}{c}\alpha \\ j\end{array}\right)$ are binomial coefficients 
$c_{j}^{(\alpha)}(j=0,1, \ldots)$. For their calculation we can use the following expressions (Dorckák, 1994; Petráš, 2010; 2011):

$$
c_{0}^{(\alpha)}=1, \quad c_{j}^{(\alpha)}=\left(1-\frac{1+\alpha}{j}\right) c_{j-1}^{(\alpha)} .
$$

Now we consider the following linear fractional-order system:

$$
\left\{\begin{array}{l}
D^{\alpha} x(t)=A x(t)+B u(t), \\
y(t)=C x(t), \\
x(0)=x_{0}
\end{array} \quad 0<\alpha<2,\right.
$$

where $x(t) \in \mathbb{R}$ is the state vector, $u(t) \in \mathbb{R}$ is the control input vector and $y(t) \in \mathbb{R}$ is the measured output. $A, B$ and $C$ are known constant matrices.

It has been shown that the system (5) is stable if the following condition is satisfied (refer to Matignon (1996; 1998) for $0<\alpha \leq 1$ and Sabatier et al. (2008) for $1<$ $\alpha<2)$ :

$$
|\arg (\operatorname{spec}(A))|>\alpha \frac{\pi}{2},
$$

where $\operatorname{spec}(A)$ represents the eigenvalues of matrix $A$.

Necessary and sufficient LMI conditions to satisfy the condition (6) if the fractional order $\alpha$ belongs to $0<$ $\alpha<2$ are given in the following two lemmas.

Lemma 1. (Sabatier et al., 2008; 2010; Chilali et al., 1999) Let $A \in \mathbb{R}^{n \times n}$. Then $|\arg (\operatorname{spec}(A))|>\alpha \pi / 2$, where $1 \leq \alpha<2$, if and only if there exits a matrix $P_{0}=$ $P_{0}^{T}>0$ such that

$$
\left[\begin{array}{cc}
\left(A P_{0}+P_{0} A^{T}\right) \sin \theta & \left(A P_{0}-P_{0} A^{T}\right) \cos \theta \\
\left(P_{0} A^{T}-A P_{0}\right) \cos \theta & \left(A P_{0}+P_{0} A^{T}\right) \sin \theta
\end{array}\right]<0,
$$

where $\theta=\pi-\alpha \pi / 2$.

Lemma 2. (Lu and Chen, 2010) Let $A \in \mathbb{R}^{n \times n}$ and $0<\alpha<1$. The fractional-order system $D^{\alpha} x(t)=A x(t)$ is asymptotically stable, i.e., $(|\arg (\operatorname{spec}(A))|>\alpha \pi / 2)$ if and only if there exist two real symmetric matrices $P_{k 1} \in$ $\mathbb{R}^{n \times n}, k=1,2$, and two skew-symmetric matrices $P_{k 2} \in$ $\mathbb{R}^{n \times n}, k=1,2$, such that

$$
\begin{gathered}
\sum_{i=1}^{2} \sum_{j=1}^{2} \operatorname{Sym}\left\{\Gamma_{i j} \otimes\left(A P_{i j}\right)\right\}<0 \\
{\left[\begin{array}{cc}
P_{11} & P_{12} \\
-P_{12} & P_{11}
\end{array}\right]>0, \quad\left[\begin{array}{cc}
P_{21} & P_{22} \\
-P_{22} & P_{21}
\end{array}\right]>0,}
\end{gathered}
$$

where

$$
\begin{aligned}
\Gamma_{11} & =\left[\begin{array}{cc}
\sin \left(\alpha \frac{\pi}{2}\right) & -\cos \left(\alpha \frac{\pi}{2}\right) \\
\cos \left(\alpha \frac{\pi}{2}\right) & \sin \left(\alpha \frac{\pi}{2}\right)
\end{array}\right], \\
\Gamma_{12} & =\left[\begin{array}{cc}
\cos \left(\alpha \frac{\pi}{2}\right) & \sin \left(\alpha \frac{\pi}{2}\right) \\
-\sin \left(\alpha \frac{\pi}{2}\right) & \cos \left(\alpha \frac{\pi}{2}\right)
\end{array}\right], \\
\Gamma_{21} & =\left[\begin{array}{cc}
\sin \left(\alpha \frac{\pi}{2}\right) & \cos \left(\alpha \frac{\pi}{2}\right) \\
-\cos \left(\alpha \frac{\pi}{2}\right) & \sin \left(\alpha \frac{\pi}{2}\right)
\end{array}\right], \\
\Gamma_{22} & =\left[\begin{array}{ll}
-\cos \left(\alpha \frac{\pi}{2}\right) & \sin \left(\alpha \frac{\pi}{2}\right) \\
-\sin \left(\alpha \frac{\pi}{2}\right) & -\cos \left(\alpha \frac{\pi}{2}\right)
\end{array}\right] .
\end{aligned}
$$

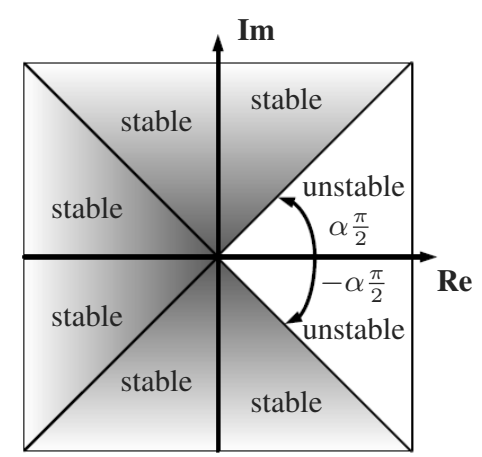

Fig. 1. Stability region of linear fractional-order systems with order $0<\alpha<1$.

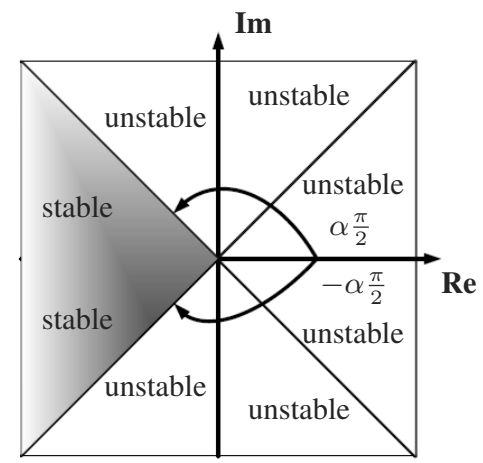

Fig. 2. Stability region of linear fractional-order systems with order $1<\alpha<2$.

The two drawings in Figs. 1 and 2 illustrate the stability regions of linear fractional-order systems with a fractional order belonging to $0<\alpha<1$ and $1 \leq \alpha<2$, respectively.

Note that the conditions presented in Lemma 2 are equivalent to those given by Sabatier et al. (2010) and Farges et al. (2010).

To prove the main results in the next section, we need the following lemmas.

Lemma 3. (Matignon and Adréa-Novel, 1996; 1997) The system (5) is detectable if and only if

$$
\operatorname{rank}\left(\left[\begin{array}{c}
\sigma I_{n}-A \\
C
\end{array}\right]\right)=n, \quad \forall \sigma \in \mathbb{C}
$$

with $|\arg (\sigma)| \leq \alpha \pi / 2$.

Proof. The result can be established as in the usual integer-order case, since it only involves algebraic properties of the pair $(C, A)$.

Remark 1. (Matignon and Adréa-Novel, 1996; 1997) In particular, if $(C, A)$ is observable, i.e.,

$$
\operatorname{rank}\left(\left[\begin{array}{c}
\sigma I_{n}-A \\
C
\end{array}\right]\right)=n, \quad \forall \sigma \in \mathbb{C},
$$


there exists a matrix gain $L$ such that the spectrum of $\mathbb{L}=$ $A-L C$ can be assigned anywhere in the complex region of asymptotic stability i.e. $(|\arg (\operatorname{spec}(\mathbb{L}))|>\alpha \pi / 2)$.

Lemma 4. (Lancaster and Tismenetsky, 1985; Ben-Israel and Greville, 1974) Let $X$ represent an $m \times n$ matrix and $Y$ an $n \times p$ matrix. Then $\operatorname{rank} X Y=\operatorname{rank} Y$ if and only if

$$
\operatorname{rank}\left[\begin{array}{c}
X \\
I-Y Y^{+}
\end{array}\right]=n .
$$

\section{Unknown input functional observer design}

In this section, we give sufficient conditions for the existence and stability of a functional observer with unknown inputs. A constructive procedure for the design of this functional observer will be presented.

Consider the following linear fractional-order system:

$$
\left\{\begin{array}{l}
D^{\alpha} x(t)=A x(t)+F d(t)+B u(t), \\
y(t)=C x(t)+G d(t), \\
z(t)=L x(t), \\
x(0)=x_{0}
\end{array}\right.
$$

where $x(t) \in \mathbb{R}^{n}$ is the state vector, $u(t) \in \mathbb{R}^{m}$ is the control input vector, $y(t) \in \mathbb{R}^{p}$ is the measured output, $d(t) \in \mathbb{R}^{q}$ is the unknown input vector and $z(t) \in \mathbb{R}^{r}$ is the vector to be estimated, where $r \leq n$. $A, B, C, F$, $G$ and $L$ are known constant real matrices of compatible dimensions.

In order to reconstruct the state function, we require a functional observer of the form

$$
\left\{\begin{array}{l}
D^{\alpha} \eta(t)=N \eta(t)+J y(t)+H u(t), \\
\widehat{z}(t)=\eta(t)+E y(t), \\
\eta(0)=\eta_{0},
\end{array}\right.
$$

where $\eta(t) \in \mathbb{R}^{r}$ is the state vector of the observer and $\widehat{z}(t) \in \mathbb{R}^{r}$ is the estimate of $z(t)$. Matrices $N, J, H$ and $E$ are unknown matrices of appropriate dimensions to be designed. Figure 3 shows the block diagram of the closed-loop fractional-order system with the fractional-order unknown input observer. Note that the fractional-order observer only uses the available input and output to reconstruct the state vector.

The following proposition gives the conditions for the existence and stability of the functional observer (12).

Proposition 1. The system (12) is an asymptotic functional observer where $0<\alpha<2$, i.e.,

$$
\lim _{t \rightarrow \infty} \widehat{z}(t)-z(t)=0
$$

for any $x(0), \widehat{z}(0)$ and $u(t)$ if

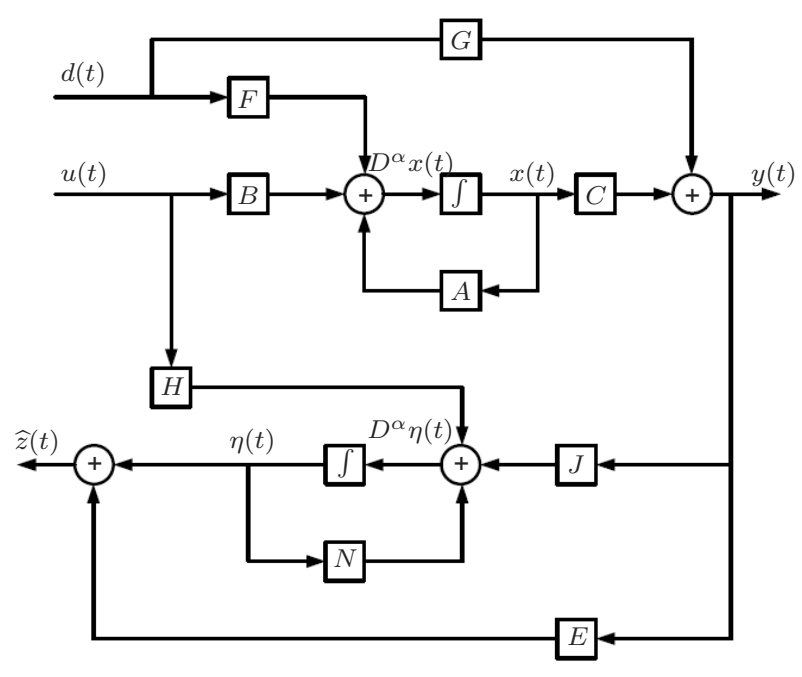

Fig. 3. Scheme of the closed-loop system with a fractionalorder unknown input observer.

(i) $D^{\alpha} e(t)=N e(t)$ is asymptotically stable,

(ii) $P A-N P-J C=0$,

(iii) $P F+N E G-J G=0$,

(iv) $H=P B$,

(v) $E G=0$,

where $P=L-E C$.

Proof. Define $e(t)=z(t)-\widehat{z}(t)$, the error between $z(t)$ and $\widehat{z}(t)$. Then its fractional-order dynamics are given by

$$
D^{\alpha} e(t)=D^{\alpha} z(t)-D^{\alpha} \widehat{z}(t)
$$

or, equivalently,

$$
\begin{aligned}
D^{\alpha} e(t)= & N e(t)+(P A-N P-J C) x(t) \\
& +(P B-H) u(t) \\
& +(P F+N E G-J G) d(t)-E G D^{\alpha} d(t) .
\end{aligned}
$$

If the conditions (i)-( $(\mathbb{V})$ are satisfied, then

$$
\lim _{t \rightarrow \infty} e(t)=0
$$

for any $x(0), \widehat{z}(0), d(t)$ and $u(t)$.

From Eqn. (14), one can see that under the conditions (ii)-(V) the fractional-order dynamics of this observer error are given by

$$
D^{\alpha} e(t)=N e(t) \quad \text { with } \quad 0<\alpha<2,
$$

and in this case $\lim _{t \rightarrow \infty} e(t)=0$ if (15) is asymptotically stable.

Now the design of the functional observer is reduced to finding matrices $N, P, J, H$ such that Proposition 1 is 
satisfied. By using the definition of $P$, the conditions (iii) and (iii) can be written as

$$
\begin{aligned}
N L+E C A+K C & =L A, \\
K G+E C F & =L F,
\end{aligned}
$$

where $K=J-N E$. Equations (16a) and 16b) and the condition (V) can be written as

$$
\left[\begin{array}{lll}
N & K & E
\end{array}\right] \Sigma_{1}=\Sigma_{2}
$$

where

$$
\Sigma_{1}=\left[\begin{array}{ccc}
L & 0 & 0 \\
C & G & 0 \\
C A & C F & G
\end{array}\right]
$$

and

$$
\Sigma_{2}=\left[\begin{array}{lll}
L A & L F & 0
\end{array}\right] .
$$

The following lemma gives necessary and sufficient conditions for the existence of a solution to 17 .

Lemma 5. There exists a solution to (17) if and only if

$$
\operatorname{rank}\left[\begin{array}{ccc}
L & 0 & 0 \\
C & G & 0 \\
C A & C F & G \\
L A & L F & 0
\end{array}\right]=\operatorname{rank}\left[\begin{array}{ccc}
L & 0 & 0 \\
C & G & 0 \\
C A & C F & G
\end{array}\right] .
$$

Proof. From the general solution of linear matrix equations (Rao and Mitra, 1971), there exists a solution of (17) if and only if

$$
\Sigma_{2} \Sigma_{1}^{+} \Sigma_{1}=\Sigma_{2},
$$

where $\Sigma_{1}^{+}$is the generalized inverse matrix of $\Sigma_{1}$. Equation (20) is equivalent to

$$
\operatorname{rank}\left[\begin{array}{c}
\Sigma_{1} \\
\Sigma_{2}
\end{array}\right]=\operatorname{rank} \Sigma_{1}
$$

which is the condition (19).

In this case the general solution of (17) is given by

$$
\left[\begin{array}{lll}
N & K & E
\end{array}\right]=\Sigma_{2} \Sigma_{1}^{+}-Z\left(I-\Sigma_{1} \Sigma_{1}^{+}\right),
$$

where $Z$ is an arbitrary matrix of appropriate dimension. From (22), we obtain

$$
N=\mathbb{A}-Z \mathbb{B},
$$

where

$$
\mathbb{A}=\Sigma_{2} \Sigma_{1}^{+}\left[\begin{array}{l}
I \\
0 \\
0
\end{array}\right]
$$

and

$$
\mathbb{B}=\left(I-\Sigma_{1} \Sigma_{1}^{+}\right)\left[\begin{array}{l}
I \\
0 \\
0
\end{array}\right]
$$

Matrices $J$ and $H$ are obtained according to

$$
\begin{gathered}
J=K+N E, \\
H=(L-E C) B .
\end{gathered}
$$

By using this algorithm, we can compute all the observer parameters which provide a fractional-order functional observer of the form (12).

Under the condition (19) and by using (23), the observer error dynamics can be written as

$$
D^{\alpha} e(t)=N e(t) \quad \text { with } \quad 0<\alpha<2 .
$$

Now, the problem of the design of the functional observer (12) is reduced to the determination of the free matrix parameter $Z$ such that condition (ii) of Proposition 1 is satisfied. The following lemma gives necessary and sufficient conditions for the existence of the matrix parameter $Z$.

Lemma 6. There exists a matrix parameter $Z$ such that (27) is asymptotically stable if and only if

$$
\operatorname{rank}\left[\begin{array}{ccc}
\sigma L-L A & -L F & 0 \\
C & G & 0 \\
C A & C F & G
\end{array}\right]=\operatorname{rank} \Sigma_{1}, \quad \forall \sigma \in \mathbb{C},
$$

with $|\arg (\sigma)| \leq \alpha \pi / 2$.

Proof. From (27), the matrix $N$ is asymptotically stable if and only if the pair $(\mathbb{B}, \mathbb{A})$ is detectable or, equivalently,

$$
\operatorname{rank}\left(\left[\begin{array}{c}
\sigma I-\mathbb{A} \\
\mathbb{B}
\end{array}\right]\right)=r, \quad \forall \sigma \in \mathbb{C}
$$

with $|\arg (\sigma)| \leq \alpha \pi / 2$.

The left-hand side of (28) can be written as

$$
\begin{gathered}
\operatorname{rank}\left[\begin{array}{ccc}
\sigma L-L A & -L F & 0 \\
C & G & 0 \\
C A & C F & G
\end{array}\right] \\
=\operatorname{rank}\left[\left[\begin{array}{ccc}
\sigma I & 0 & 0 \\
0 & I & 0 \\
0 & 0 & I
\end{array}\right] \Sigma_{1}-\left[\begin{array}{c}
\Sigma_{2} \\
0
\end{array}\right]\right] \\
=\operatorname{rank}\left[\left[\begin{array}{ccc}
\sigma I & 0 & 0 \\
0 & I & 0 \\
0 & 0 & I
\end{array}\right]-\left[\begin{array}{c}
\Sigma_{2} \Sigma_{1}^{+} \\
0
\end{array}\right]\right] \Sigma_{1} .
\end{gathered}
$$

From this equality one can see that 28 is satisfied if and only if

$$
\operatorname{rank}\left[\left[\begin{array}{ccc}
\sigma I & 0 & 0 \\
0 & I & 0 \\
0 & 0 & I
\end{array}\right]-\left[\begin{array}{c}
\Sigma_{2} \Sigma_{1}^{+} \\
0
\end{array}\right]\right] \Sigma_{1}=\operatorname{rank} \Sigma_{1}
$$


$\forall \sigma \in \mathbb{C}$ with $|\arg (\sigma)| \leq \alpha \pi / 2$.

Using Lemma 4, this is equivalent to the condition that

$$
\left[\begin{array}{c}
{\left[\begin{array}{ccc}
\sigma I & 0 & 0 \\
0 & I & 0 \\
0 & 0 & I
\end{array}\right]-\left[\begin{array}{c}
\Sigma_{2} \Sigma_{1}^{+} \\
0
\end{array}\right]} \\
I-\Sigma_{1} \Sigma_{1}^{+}
\end{array}\right]
$$

is of full column rank $\sigma \in \mathbb{C}$ and $|\arg (\sigma)| \leq \alpha \pi / 2$.

Or, equivalently, the matrix

$$
\left[\begin{array}{ccc}
\sigma I-\mathbb{A} & -\Sigma_{2} \Sigma_{1}^{+}\left[\begin{array}{l}
0 \\
I \\
0
\end{array}\right] & -\Sigma_{2} \Sigma_{1}^{+}\left[\begin{array}{l}
0 \\
0 \\
I
\end{array}\right] \\
\mathbb{B} & \left(I-\Sigma_{1} \Sigma_{1}^{+}\right)\left[\begin{array}{l}
0 \\
I \\
0
\end{array}\right] & \left(I-\Sigma_{1} \Sigma_{1}^{+}\right)\left[\begin{array}{l}
0 \\
0 \\
I
\end{array}\right] \\
0 & I & 0 \\
0 & 0 & I
\end{array}\right]
$$

must be of full column rank, $\forall \sigma \in \mathbb{C}$ and $|\arg (\sigma)| \leq$ $\alpha \pi / 2$. This is equivalent to

$$
\operatorname{rank}\left[\begin{array}{c}
\sigma I-\mathbb{A} \\
\mathbb{B}
\end{array}\right]=r, \quad \forall \sigma \in \mathbb{C}
$$

with $|\arg (\sigma)| \leq \alpha \pi / 2$, which ends the proof.

The asymptotical stability for the fractional-order observer error system (27) where $0<\alpha \leq 1$ and $1 \leq$ $\alpha<2$ is given in the two following theorems.

Theorem 1. Under the conditions (19) and (28), there exists an asymptotically stable observer of the form (12) where $1 \leq \alpha<2$ if there are matrices $X \in \mathbb{R}^{m \times n}$ and $P_{0}=P_{0}^{T}>0 \in \mathbb{R}^{n \times n}$ such that

$$
\left[\begin{array}{ll}
\Omega_{11} & \Omega_{12} \\
\Omega_{12}^{T} & \Omega_{22}
\end{array}\right]<0
$$

where

$$
\begin{aligned}
& \Omega_{11}=\Omega_{22}=\left(P_{0} \mathbb{A}+\mathbb{A}^{T} P_{0}-X \mathbb{B}-\mathbb{B}^{T} X^{T}\right) \sin \theta, \\
& \Omega_{12}=\left(\mathbb{A}^{T} P_{0}-P_{0} \mathbb{A}+X \mathbb{B}-\mathbb{B}^{T} X^{T}\right) \cos \theta .
\end{aligned}
$$

Moreover, a stabilizing gain matrix $Z$ is given by

$$
Z=X P_{0}^{-1}
$$

Proof. From Lemmas 3 and 6, one can see that a necessary condition in order to satisfy the condition (ii) of Proposition 1 by using (23), implies that the pair $(\mathbb{B}, \mathbb{A})$ is detectable. Now, suppose that there exist matrices $X \in$ $\mathbb{R}^{m \times n}$ and $P_{0}=P_{0}^{T}>0 \in \mathbb{R}^{n \times n}$ such that (30) holds. It follows from Lemma 1 that $|\arg (\operatorname{spec}(N))|>\alpha \pi / 2$ is equivalent to

$$
\begin{aligned}
& {\left[\begin{array}{cc}
\left(P_{0} N+N^{T} P_{0}\right) \sin \theta & -\left(P_{0} N-N^{T} P_{0}\right) \cos \theta \\
\left(P_{0} N-N^{T} P_{0}\right) \cos \theta & \left(P_{0} N+N^{T} P_{0}\right) \sin \theta
\end{array}\right]} \\
& =\operatorname{Sym}\left\{\left[\begin{array}{cc}
P_{0} \mathbb{A} \sin \theta & -P_{0} \mathbb{A} \cos \theta \\
P_{0} \mathbb{A} \cos \theta & P_{0} \mathbb{A} \sin \theta
\end{array}\right]\right\} \\
& +\operatorname{Sym}\left\{\left[\begin{array}{cc}
-X \mathbb{B} \sin \theta & X \mathbb{B} \cos \theta \\
-X \mathbb{B} \cos \theta & -X \mathbb{B} \sin \theta
\end{array}\right]\right\}<0,
\end{aligned}
$$

where $Z=X P_{0}^{-1}$ and $\theta=\pi-\alpha \pi / 2$. The inequality (31) is equivalent to (30). This ends the proof.

Theorem 2. Under the conditions (19) and (28), there exists an asymptotically stable observer of the form (12) where $0<\alpha \leq 1$ if and only if there are matrices $Q \in$ $\mathbb{R}^{m \times n}$ and $P_{0}=P_{0}^{T}>0 \in \mathbb{R}^{n \times n}$ such that

$\sum_{i=1}^{2}\left(\operatorname{Sym}\left\{\Gamma_{i 1} \otimes\left(\mathbb{A}^{T} P_{0}\right)\right\}-\operatorname{Sym}\left\{\Gamma_{i 1} \otimes\left(\mathbb{B}^{T} Q\right)\right\}\right)<0$,

where $\Gamma_{i 1}(i=1,2)$ satisfy (9) and the stabilizing gain matrix $Z$ is given by $Z=P_{0}^{-1} Q^{T}$.

Proof. From Lemmas 3 and 6, one can see that a necessary condition in order for the condition (ii) of Proposition 1 be satisfied, by using (23), implies that the pair $(\mathbb{B}, \mathbb{A})$ is detectable. Suppose that there exist matrices $Q \in \mathbb{R}^{m \times n}$ and $P_{0}=P_{0}^{T}>0 \in \mathbb{R}^{n \times n}$ such that (32) holds. It follows from Lemma 2 that $|\arg (\operatorname{spec}(N))|>\alpha \pi / 2$ is equivalent to

$$
\sum_{i=1}^{2} \sum_{j=1}^{2} \operatorname{Sym}\left\{\Gamma_{i j} \otimes\left(N^{T} P_{i j}\right)\right\}<0
$$

where $N=\mathbb{A}-Z \mathbb{B}$ and $\Gamma_{i j}(i, j=1,2)$ satisfy (9). By setting $P_{11}=P_{21}=P_{0}, P_{12}=P_{22}=0$ in (33), one can conclude that, if

$$
\operatorname{Sym}\left\{\Gamma_{11} \otimes\left(N^{T} P_{0}\right)\right\}+\operatorname{Sym}\left\{\Gamma_{21} \otimes\left(N^{T} P_{0}\right)\right\}<0,
$$

the fractional-order system $D^{\alpha} e(t)=N e(t)$, where $0<$ $\alpha \leq 1$, is asymptotically stable. Substitution of $N=$ $\mathbb{A}-Z \mathbb{B}$ into (34) and setting $Z=P_{0}^{-1} Q^{T}$ result in

$$
\sum_{i=1}^{2}\left(\operatorname{Sym}\left\{\Gamma_{i 1} \otimes\left(\mathbb{A}^{T} P_{0}\right)\right\}-\operatorname{Sym}\left\{\Gamma_{i 1} \otimes\left(\mathbb{B}^{T} Q\right)\right\}\right)<0 .
$$

The inequality (35) is equivalent to (32). This completes the proof.

The following design algorithm can be carried out for the design of a fractional-order observer. 
Algorithm 1. Design.

Step 1. Check if (19) in Lemma5 is satisfied or not. If not, STOP as a fractional-order observer does not exist.

Step 2. From (24), obtain matrices $\mathbb{A}$ and $\mathbb{B}$. Check if the pair $(\mathbb{B}, \mathbb{A})$ is detectable. If not, STOP, as a stable fractional-order observer does not exist.

Step 3. Use (23) to derive $Z$ such that matrix $N$ is stable.

Step 4. From (22), obtain matrices $N, K$ and $E$.

Step 5. Obtain matrix $P$, where $P=L-E C$.

Step 6. Use (25) to obtain matrix $J$. Finally, obtain matrix $H$ from (26). The fractional-order observer design is thus completed.

\section{Numerical examples}

In this section, we provide two numerical examples to illustrate the applicability of the proposed method.

Example 1. $(\alpha=1.76)$ Consider the linear fractional-order system (11) with the following matrices:

$$
\begin{aligned}
A & =\left[\begin{array}{cccc}
-1 & 0 & 0 & 0 \\
1 & -2 & 1 & 0 \\
0 & 1 & -3 & 0 \\
0 & 0 & 0 & -4
\end{array}\right], & F=\left[\begin{array}{l}
1 \\
0 \\
0 \\
0
\end{array}\right], & \\
B & =\left[\begin{array}{l}
0 \\
1 \\
0 \\
1
\end{array}\right], & & C=\left[\begin{array}{llll}
1 & 0 & 0 & 0 \\
0 & 1 & 0 & 0
\end{array}\right], \\
L & =\left[\begin{array}{llll}
0 & 0 & 1 & 0
\end{array}\right], & G & =\left[\begin{array}{l}
0 \\
0
\end{array}\right] .
\end{aligned}
$$

One can see that the conditions (19) and (28) are satisfied.

From the results of Section 3 , we obtain

$$
\mathbb{A}=-2.2857,
$$

$$
\mathbb{B}=\left[\begin{array}{lllll}
0.1429 & 0.1429 & -0.2857 & 0 & -0.1429
\end{array}\right]^{T} .
$$

A feasible solution of the LMI (30), where $\alpha=1.76$, is as follows:

$$
\begin{gathered}
P_{0}=49.402, \\
X=\left[\begin{array}{lllll}
3.0876 & 3.0876 & -6.1752 & 0 & -3.0876
\end{array}\right] .
\end{gathered}
$$

Then, the asymptotically stabilizing state-feedback gain is obtained as

$$
\begin{aligned}
Z & =X P_{0}^{-1} \\
& =\left[\begin{array}{lllll}
0.0625 & 0.0625 & -0.1250 & 0 & -0.0625
\end{array}\right] .
\end{aligned}
$$

Using Algorithm 1, we obtain the following functional observer parameters:

$$
\begin{aligned}
N & =-2.3482, & K & =\left[\begin{array}{lll}
0.6518 & -0.3036
\end{array}\right], \\
E & =\left[\begin{array}{llll}
0 & -0.6518
\end{array}\right], & J & =\left[\begin{array}{lll}
0.6518 & 1.2270
\end{array}\right], \\
P & =\left[\begin{array}{llll}
0 & 0.6518 & 1 & 0
\end{array}\right], & H & =0.6518 .
\end{aligned}
$$

Finally, the estimate $\widehat{z}(t)$ is given by the following observer:

$$
\left\{\begin{aligned}
& D^{\alpha} \eta(t)=-2.3482 \eta(t)+\left[\begin{array}{ll}
0.6518 & 1.2270
\end{array}\right] y(t) \\
&+0.6518 u(t) \\
& \widehat{z}(t)=\eta(t)-0.6518 y_{2}(t)
\end{aligned}\right.
$$

with $\alpha=1.76$

Figures 3 and 4 show the performance of the functional observer presented in this paper for $\alpha=1.76$ and $\alpha=1$ with the unknown input vector $d(t)=$ $0.5 \sin (60 \pi t)$.

The simulation for the proposed fractional-order observer was performed with $\widehat{z}_{0}=800$. Figures 3 and 4 show the true and estimated trajectories of the state $z(t)$ and the corresponding estimation error. These simulation results demonstrate that our proposed design is effective.

Example 2. $(\alpha=0.77)$ Consider the linear fractional-order system (11) with the following matrices:

$$
\begin{aligned}
A & =\left[\begin{array}{cccc}
-5 & 0 & 0 & 0 \\
3 & -8 & 1 & 0 \\
0 & -1 & -10 & 0 \\
0 & 0 & 0 & -7
\end{array}\right], & & \\
F & =\left[\begin{array}{l}
1 \\
0 \\
2 \\
1
\end{array}\right], & B=\left[\begin{array}{l}
1 \\
1 \\
0 \\
1
\end{array}\right], & C=\left[\begin{array}{cccc}
-1 & 0 & 0 & 0 \\
0 & 2 & 0 & 0
\end{array}\right], \\
L & =\left[\begin{array}{llll}
0 & 0 & 1 & 0
\end{array}\right], & G=\left[\begin{array}{l}
0 \\
0
\end{array}\right] . &
\end{aligned}
$$

It can easily be seen that the conditions (19) and (28) are satisfied.

From the results of Section 3, we obtain

$$
\mathbb{A}=-10.8381
$$

$$
\mathbb{B}=\left[\begin{array}{lllll}
0.0381 & -0.1143 & -0.1524 & 0 & -0.0190
\end{array}\right]^{T} .
$$

A feasible solution of the LMI (32), where $\alpha=0.77$, is as follows:

$$
P_{0}=243.98,
$$

$$
Q=\left[\begin{array}{lllll}
0.8576 & -2.5738 & -3.4303 & 0 & -0.4288
\end{array}\right]^{T} .
$$

Then, the asymptotically stabilizing state-feedback gain is obtained as

$$
\begin{aligned}
Z & =P_{0}^{-1} Q^{T} \\
& =\left[\begin{array}{llllll}
0.0035 & -0.0105 & -0.0141 & 0 & -0.0018
\end{array}\right] .
\end{aligned}
$$

Using Algorithm 1, we obtain the following functional 
observer parameters:

$$
\begin{aligned}
& N=-10.8416, \\
& K=\left[\begin{array}{ll}
-7.4752 & 2.8664
\end{array}\right] \text {, } \\
& E=\left[\begin{array}{ll}
-2 & 0.4208
\end{array}\right] \text {, } \\
& J=\left[\begin{array}{ll}
14.2080 & -1.6957
\end{array}\right], \\
& P=\left[\begin{array}{llll}
-2 & -0.8416 & 1 & 0
\end{array}\right] \text {, } \\
& H=-2.8416 \text {. }
\end{aligned}
$$

Finally, the estimate $\widehat{z}(t)$ is given by the following fractional-order observer:

$$
\left\{\begin{array}{c}
D^{\alpha} \eta(t)=-10.8416 \eta(t)+\left[\begin{array}{ll}
14.2080 & -1.6957
\end{array}\right] y(t) \\
\quad-2.8416 u(t), \\
\widehat{z}(t)=\eta(t)+\left[\begin{array}{ll}
-2 & 0.4208
\end{array}\right] y(t),
\end{array}\right.
$$

where $\alpha=0.77$.

Figures 5 and 6 show the performance of the functional observer presented in this paper for $\alpha=0.77$ and $\alpha=1$ with the unknown input vector

$$
d(t)=\left[\begin{array}{l}
0.25 \sin (40 \pi t) \\
0.45 \sin (40 \pi t)
\end{array}\right]
$$

\section{Conclusion}

In this paper, we presented a simple method to design a functional observer for linear fractional-order systems. This method reduces the design procedure to one such as known from integer-order systems. Some conditions for the existence of these observers are given, and sufficient conditions for their stability are derived in terms of linear matrix inequalities for a fractional order satisfying $0<$ $\alpha<2$. It is shown that the fractional-order observer design is as stable as its integer-order counterpart and guarantees the convergence of the estimation error. Two illustrative examples have shown the effectiveness of our results.

\section{References}

Bagley, R. and Calico, R. (1991). Fractional order state equations for the control of viscoelastically damped structures, Journal of Guidance, Control, and Dynamics 14(2): 304-311.

Ben-Israel, A. and Greville, T.N.E. (1974). Generalized Inverses: Theory and Applications, Wiley, New York, NY.

Boroujeni, E.A. and Momeni, H.R. (2012). Non-fragile nonlinear fractional order observer design for a class of nonlinear fractional order systems, Signal Processing 92(10): 2365-2370.

Boutayeb, M., Darouach, M. and Rafaralahy, H. (2002). Generalized state-space observers for chaotic synchronization and secure communication, IEEE Transactions on Circuits and Systems, I: Fundamental Theory and Applications 49(3): 345-349.

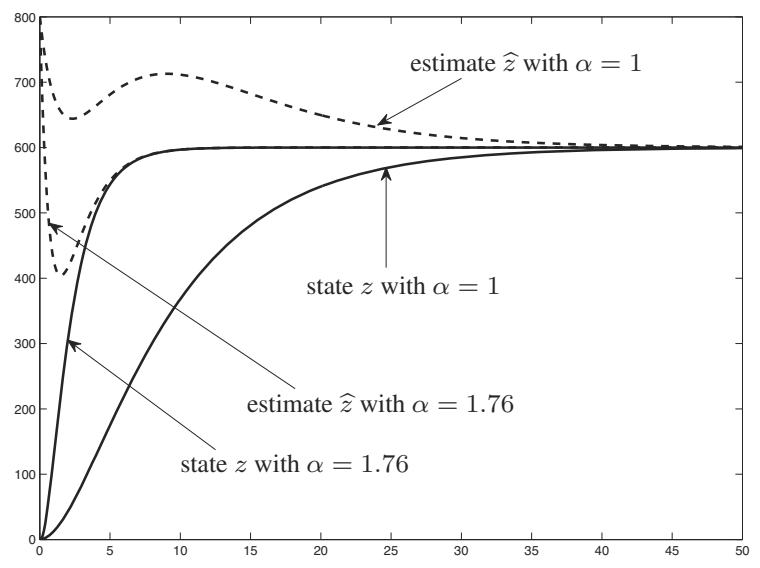

Fig. 4. State response $z$ and its estimate $\widehat{z}$ in Example 1 with fractional orders $\alpha=1.76$ and $\alpha=1$.

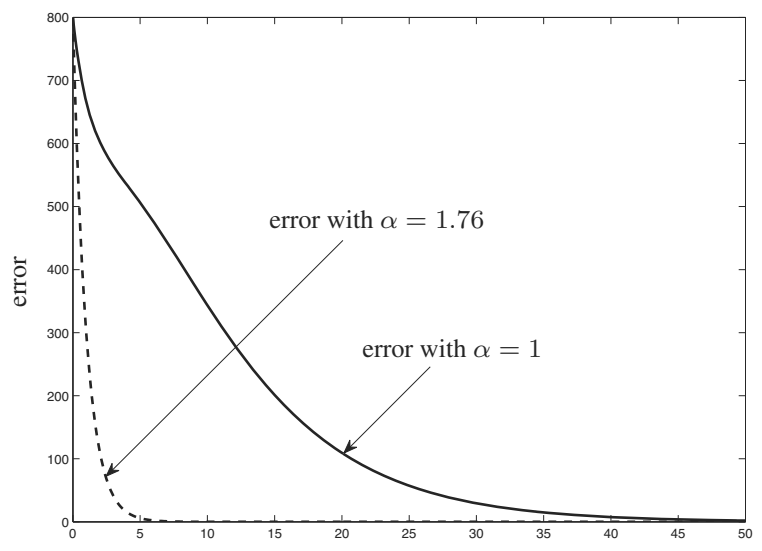

Fig. 5. Error function of the functional observer in Example 1 with fractional orders $\alpha=1.76$ and $\alpha=1$.

Caponetto, R., Dongola, G., Fortuna, L. and Petráš, I. (2010). Fractional Order Systems: Modeling and Control Applications, World Scientific Series on Nonlinear Science, Series A, World Scientific, Singapore.

Chen, Y., Ahn, H. and Podlubny, I. (2006). Robust stability check of fractional order linear time invariant systems with interval uncertainties, Signal Processing 86(10): 2611-2618.

Chen, Y., Vinagre, B.M. and Podlubny, I. (2004). Fractional order disturbance observer for robust vibration suppression, Nonlinear Dynamics 38(1): 355-367.

Chilali, M., Gahinet, P. and Apkarian, P. (1999). Robust pole placement in LMI regions, IEEE Transactions on Automatic Control 44(12): 2257-2270.

Dadras, S. and Momeni, H. (2011a). A new fractional order observer design for fractional order nonlinear systems, ASME 2011 International Design Engineering Technical Conference \& Computers and Information in Engineering Conference, Washington, DC, USA, pp. 403-408.

Dadras, S. and Momeni, H.R. (2011b). Fractional sliding mode observer design for a class of uncertain fractional order 


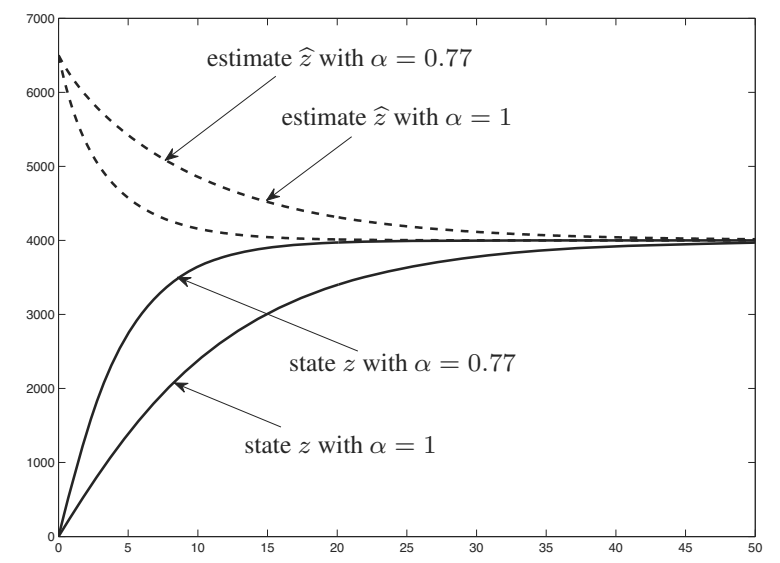

Fig. 6. State response $z$ and its estimate $\widehat{z}$ in Example 2 with fractional orders $\alpha=0.77$ and $\alpha=1$.

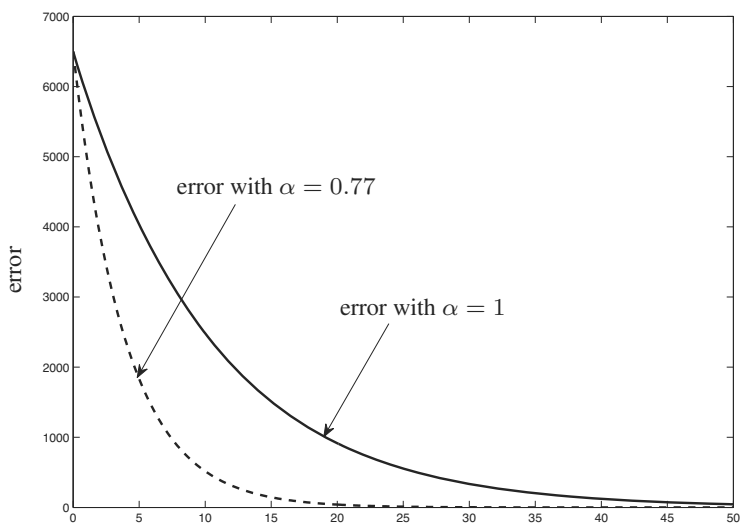

Fig. 7. Error function of the functional observer in Example 2 with fractional orders $\alpha=0.77$ and $\alpha=1$.

nonlinear systems, IEEE Conference on Decision \& Control, Orlando, FL, USA, pp. 6925-6930.

Darouach, M. (2000). Existence and design of functional observers for linear systems, IEEE Transactions on Automatic Control 45(5): 940-943.

Darouach, M., Zasadzinski, M. and Xu, S. (1994). Full-order observers for linear systems with unknown inputs, IEEE Transactions on Automatic Control 39(3): 606-609.

Delshad, S.S., Asheghan, M.M. and Beheshti, M.M. (2011). Synchronization of n-coupled incommensurate fractional-order chaotic systems with ring connection, Communications in Nonlinear Science and Numerical Simulation 16(9): 3815-3824.

Deng, W. (2007). Short memory principle and a predictor-corrector approach for fractional differential equations, Journal of Computational and Applied Mathematics 206(1): 174-188.

Dorckák, L. (1994). Numerical models for simulation the fractional-order control systems, Technical Report UEF04-94, Slovak Academy of Sciences, Kosice.
Engheta, N. (1996). On fractional calculus and fractional multipoles in electromagnetism, IEEE Transactions on Antennas and Propagation 44(4): 554-566.

Farges, C., Moze, M. and Sabatier, J. (2010). Pseudo-state feedback stabilization of commensurate fractional order systems, Automatica 46(10): 1730-1734.

Heaviside, O. (1971). Electromagnetic Theory, 3rd Edn., Chelsea Publishing Company, New York, NY.

Hilfer, R. (2001). Applications of Fractional Calculus in Physics, World Scientific Publishing, Singapore.

Kaczorek, T. (2011a). Selected Problems of Fractional Systems Theory, Lecture Notes in Control and Information Sciences, Vol. 411, Springer-Verlag, Berlin.

Kaczorek, T. (2011b). Singular fractional linear systems and electrical circuits, International Journal of Applied Mathematics and Computer Science 21(2): 379-384, DOI: 10.2478/v10006-011-0028-8.

Kilbas, A., Srivastava, H. and Trujillo, J. (2006). Theory and Applications of Fractional Differential Equations, North-Holland Mathematics Studies, Vol. 204, Elsevier, Amsterdam.

Lancaster, P. and Tismenetsky, M. (1985). The Theory of Matrices, 2nd Edn., Academic Press, Orlando, FL.

Lu, J. and Chen, Y. (2010). Robust stability and stabilization of fractional-order interval systems with the fractional-order $\alpha$ : The $0<\alpha<1$ case, IEEE Transactions on Automatic Control 55(1): 152-158.

Matignon, D. (1996). Stability results for fractional differential equations with applications to control processing, IEEE International Conference on Systems, Man, Cybernetics, Lille, France, pp. 963-968.

Matignon, D. (1998). Generalized fractional differential and difference equations: Stability properties and modelling issues, Mathematical Theory of Networks and Systems Symposium, Padova, Italy, pp. 503-506.

Matignon, D. and Andréa-Novel, B. (1996). Some results on controllability and observability of finite-dimensional fractional differential systems, Mathematical Theory of Networks and Systems Symposium, Lille, France, pp. 952-956.

Matignon, D. and Andréa-Novel, B. (1997). Observer-based for fractional differential systems, IEEE Conference on Decision and Control, San Diego, CA, USA, pp. 4967-4972.

Monje, C.A., Chen, Y.Q., Vinagre, B.M., Xue, D. and Feliu, V. (2010). Fractional-order Systems and Controls: Fundamentals and Applications, Springer, Berlin.

Petrás̆, I. (2010). A note on the fractional-order Volta system, Communications in Nonlinear Science and Numerical Simulation 15(2): 384-393.

Petrás̆, I. (2011). Fractional-Order Nonlinear Systems: Modeling, Analysis and Simulation, Springer, Berlin.

Petráš, I., Chen, Y. and Vinagre, B. (2004). Robust stability test for interval fractional-order linear systems, in $\mathrm{V}$. Blondel and A. Megretski (Eds.), Unsolved Problems in the Mathematics of Systems and Control, Vol. 38, Princeton University Press, Princeton, NJ, pp. 208-210. 
Podlubny, I. (1999). Fractional Differential Equations, Academic, New York, NY.

Podlubny, I. (2002). Geometric and physical interpretation of fractional integration and fractional differentiation, Fractional Calculus \& Applied Analysis 5(4): 367-386.

Rao, C. and Mitra, S. (1971). Generalized Inverse of Matrices and Its Applications, Wiley, New York, NY.

Rossikhin, Y. and Shitikova, M. (1997). Application of fractional derivatives to the analysis of damped vibrations of viscoelastic single mass system, Acta Mechanica 120(109): 109-125.

Sabatier, J., Farges, C., Merveillaut, M. and Feneteau, L. (2012). On observability and pseudo state estimation of fractional order systems, European Journal of Control 18(3): 260-271.

Sabatier, J., Moze, M. and Farges, C. (2008). On stability of fractional order systems, IFAC Workshop on Fractional Differentiation and Its Application, Ankara, Turkey.

Sabatier, J., Moze, M. and Farges, C. (2010). LMI conditions for fractional order systems, Computers \& Mathematics with Applications 59(5): 1594-1609.

Sun, H., Abdelwahad, A. and Onaral, B. (1984). Linear approximation of transfer function with a pole of fractional order, IEEE Transactions on Automatic Control 29(5): 441-444.

Trigeassou, J., Maamri, N., Sabatier, J. and Oustaloup, A. (2011). A Lyapunov approach to the stability of fractional differential equations, Signal Processing 91(3): 437-445.

Trinh, H. and Fernando, T. (2012). Functional Observers for Dynamical Systems, Lecture Notes in Control and Information Sciences, Vol. 420, Springer, Berlin.

Tsui, C. (1985). A new algorithm for the design of multifunctional observers, IEEE Transactions on Automatic Control 30(1): 89-93.

Van Dooren, P. (1984). Reduced-order observers: A new algorithm and proof, Systems \& Control Letters 4(5): 243-251.

Watson, J. and Grigoriadis, K. (1998). Optimal unbiased filtering via linear matrix inequalities, Systems \& Control Letters 35(2): 111-118.

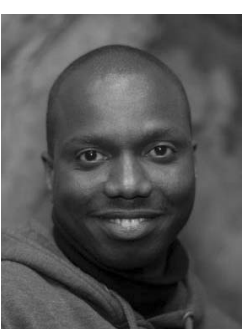

Ibrahima N'Doye received his Ph.D. degree in automatic control from the Henri Poincaré University of Nancy at the Research Center of Automatic Control (CRAN-CNRS, University of Lorraine), France, and Hassan II Ain Chock University, Casablanca, Morocco, in 2011. He is presently a postdoc in the Research Unit in Engineering Science (RUES) at the University of Luxembourg. His research interests are in estimation and control of fractional-order systems and nonlinear dynamic systems with applications in biomedicine.

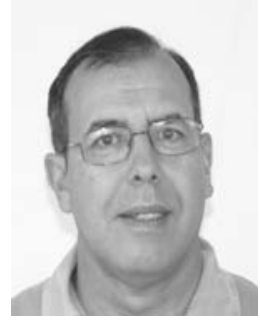

Mohamed Darouach graduated from Mohammadia Engineering School, Rabat, Morocco, in 1978, and received the Docteur Ingénieur and Doctor of Sciences degrees from Nancy University, France, in 1983 and 1986, respectively. From 1978 to 1986 he was an associate professor and a professor of automatic control at the Hassania School of Public Works, Casablanca, Morocco. Since 1987 he has been a professor at the University of Lorraine. He was a vice-director of the Research Center in Automatic Control of Nancy (CRAN UMR 7039, Nancy University, CNRS) from 2005 to 2013. Since 2010 he has been a member of the scientific council of Luxembourg University. He has held invited positions at the University of Alberta, Edmonton. His research interests include theoretical control, observer design, and control of large-scale uncertain systems with applications.

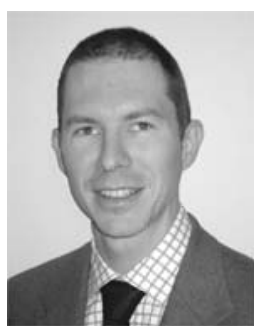

Holger Voos studied electrical engineering at Saarland University and received a doctoral degree in automatic control from the Technical University of Kaiserslautern, Germany, in 2002. From 2000 to 2004, he was with Bodenseewerk Gerätetechnik $\mathrm{GmbH}$, Germany, where he worked as a systems engineer in aerospace and robotics. From 2004 to 2010, he was a professor at the University of Applied Sciences Ravensburg-Weingarten, Germany, and the head of the Mobile Robotics Lab there. Since 2010, he has been a professor at the University of Luxembourg in the Faculty of Science, Technology and Communication, Research Unit of Engineering Sciences. He is the head of the Automatic Control Research Group and of the Automation Lab at the Interdisciplinary Centre of Security, Reliability and Trust (SnT) at the University of Luxembourg. His research interests are in the area of distributed and networked control, model predictive control, and safe and secure automation systems with applications in mobile robotics, energy systems and biomedicine.

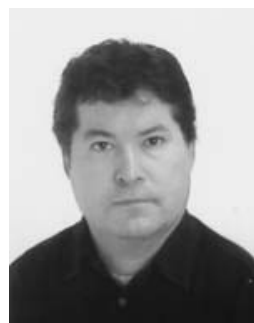

Michel Zasadzinski received his Ph.D. degree in automatic control from Nancy University, France, in 1990. He was an assistant professor at Henri Poincaré University and, from 1992 to 2000, he was a CNRS researcher in the Research Center for Automatic Control of Nancy (CRAN, CNRS). Michel Zasadzinski is now a professor at the University Institute of Technology (Longwy, University of Lorraine (former Nancy University)). His research interests encompass the theory and application of robust control and filtering for linear and nonlinear systems and for stochastic differential equations.

Received: 15 June 2012

Revised: 19 February 2013 